\title{
Der Weg zur gezielten Abgeltung medizinischen Fortschritts
}

\section{Mirjam Bach}

Dr. med., Expertin, FMH

Die Medizin ist keine in Stein gemeisselte Wissenschaft. Fortschritte etablieren sich primär im klinischen Alltag. Entsprechend anpassungsfähig muss sich das Abrechnungssystem präsentieren. Das Beispiel der neuen Sepsisdefinition zeigt, wie sich eine klinische Entwicklung dank der Initiative der FMH und partnerschaftlicher Kooperation kohärent tarifarisch abbilden lässt.

Die Definition der Sepsis wurde bereits mehrfach adaptiert. Die letzte Anpassung der Sepsisdefinition stammt aus dem Jahr 2001 (Sepsis 2). Diese Definition der Sepsis als Infektion mit mindestens 2 von 4 Kriterien des Systemischen Inflammatorischen ResponseSyndroms (SIRS) erwies sich zur Diagnosestellung allerdings zunehmend als unzureichend spezifisch bei ausgeprägter Sensitivität. Zusätzlich ermöglichen wissenschaftliche Erkenntnisse, die den zellulären Metabolismus betreffen, heute ein neues Sepsisverständnis. Die körpereigene Reaktion und die daraus resultierende Organdysfunktion gelten dabei als essentiell. Ein septischer Schock beinhaltet kreislaufwirksame und zellulär-metabolische Veränderungen. Besagte Vorgänge sind entscheidend für eine höhere Letalität und folgenschwerer als lediglich eine therapierefraktäre Hypotonie. Diese neuen Erkenntnisse erforderten eine neue Definition. Die Konsensuskonferenz definierte 2014 in der Folge die Kriterien für die Sepsis-3 wie folgt: Infektion - vermutet oder bestätigt - in Begleitung einer Organdysfunktion mit mindestens zwei Punkten des SOFA(Sequential-Organ-Failure-Assessment)Scors. Diese neue Auslegung der Sepsis zielt auf eine schnellere und präzisere Identifizierung von Risikopatienten und schafft grössere Behandlungsspielräume. Der SOFA-Score setzt sich wie folgt zusammen:

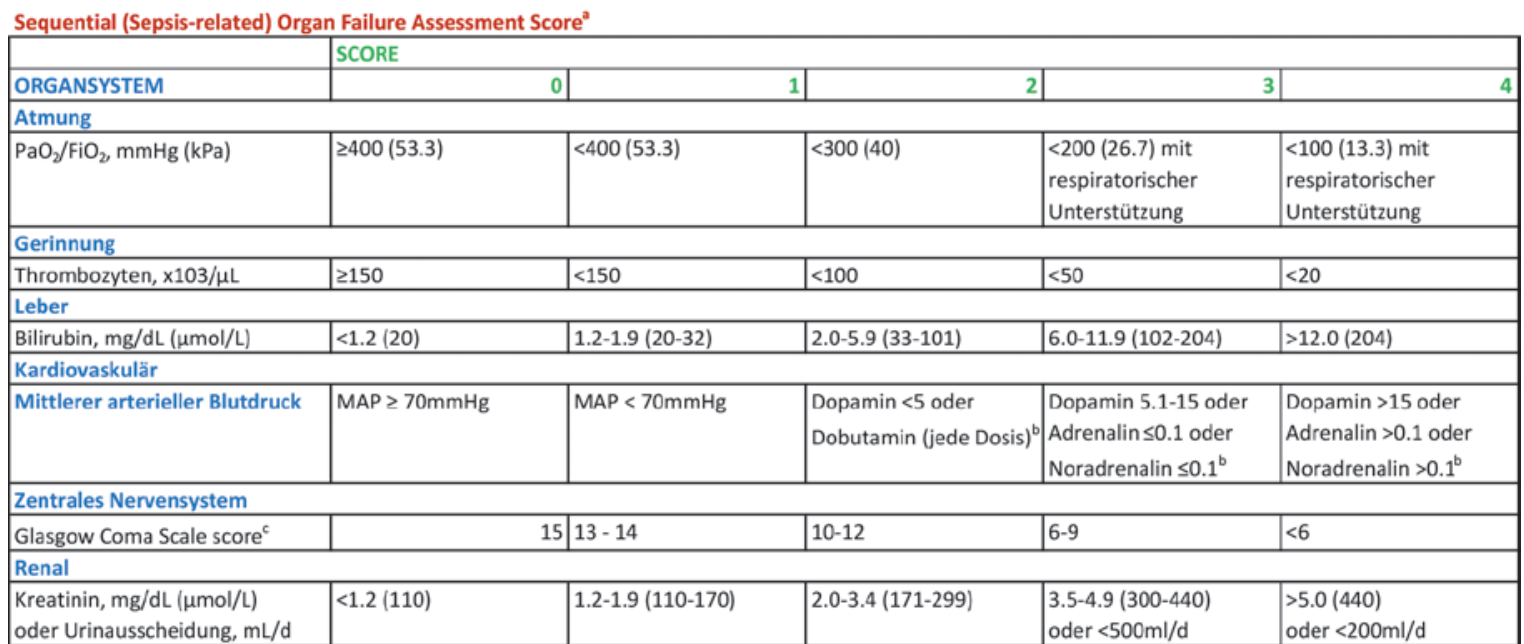

Fussnote

${ }^{2}$ Adaptiert von Vincent et $\mathrm{Al}^{27}$

berabreichte Katecholamin-Dosen in $\mu \mathrm{g} / \mathrm{kg} / \mathrm{min}$ für mindestens eine Stunde

'Glasgow Coma Scale Score reicht von 3-15

${ }^{27}$ Vincent JL, Moreno R, Takala J, et al; Working Group on Sepsis-Related Problems of the European Society of Intensive Care Medicine. The SOFA (SepsisQuelle: JAMA. 2016;315(8):801-8101 


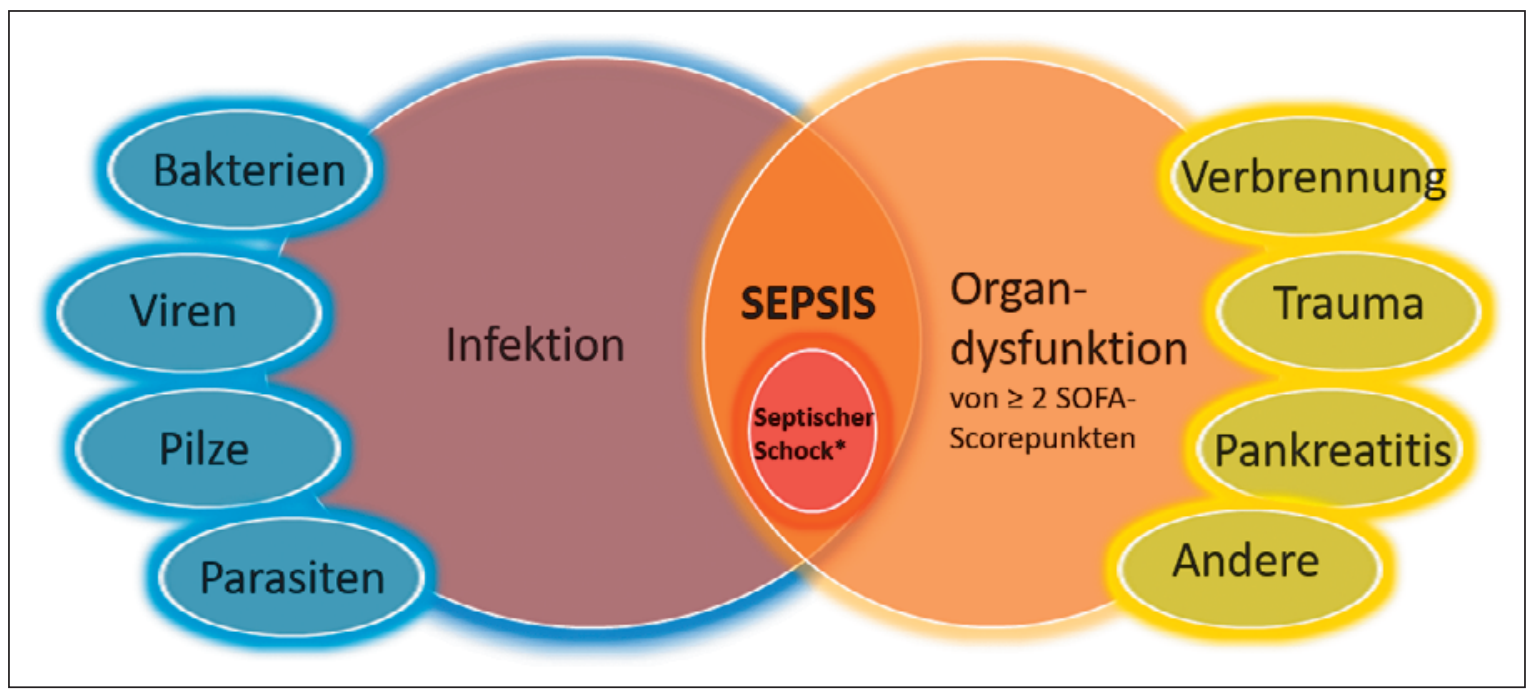

Zusammenhang zwischen Infektion, Sepsis und Organdysfunktion bei Sepsis-3 (Quelle: Deutsches Aerzteblatt, adaptiert von Weis et al. [1]); * bedingt eine volumenresistente Hypotonie und eine Laktatserumkonzentration von mehr als 2 mmol/l nach adäquater Volumensubstitution.

Im klinischen Alltag setzt sich die neue Sepsisdefinition zunehmend durch. Entsprechend gilt es, diesen klinischen Fortschritt auch im Tarifsystem abzubilden.

\section{Die Abbildung der Sepsis in der Kodierung}

Die aufwandsgerechte Abbildung eines klinischen Falles im Tarifsystem erfordert ein zielorientiertes $\mathrm{Zu}$ sammenspiel aller Beteiligten. Im stationären Bereich

Eine aufwandsgerechte Abbildung von Sepsisfällen ist nur bei Kongruenz der Kodiergrundlagen möglich.

passt die SwissDRG AG datenbasiert jährlich das Fallpauschalensystem SwissDRG an. Eine Gruppierungssoftware nimmt die Einteilung eines klinischen Falles in die entsprechende diagnosebasierte Fallgruppe (DRG) vor. Dabei erfolgt die systematische Abfrage verschiedener Parameter und die Zuteilung der Fallschwere anhand der erfassten Hauptdiagnose, Nebendiagnosen und Behandlungen. Hier kommt die Rolle eines weiteren Players zum Tragen: Das Bundesamt für Statistik (BFS) publiziert die in der Schweiz gültigen Klassifikationskataloge für Diagnosen (ICD-10-GM) und Behandlungen (CHOP). Mit der Erstellung der Kodierrichtlinien obliegt dem BFS die Anwendungsregelung der Klassifikationssysteme für die Gruppierungssoftware. Eine spezielle Kodierrichtlinie regelt im Kodierungshandbuch, wie eine Sepsis zu kodieren ist. Folgende Diagnosekombination ist für eine korrekte Fallzuordnung erforderlich:
- Diagnose, die den Infektfokus benennt

- «Sepsis durch den verursachenden Keim»

- SIRS mit oder ohne Organkomplikation

- Gegebenenfalls resultierende, behandelte Organdysfunktionen

Die Kodierung eines infektiösen SIRS mit Organkomplikation (ICD-10-Code: R65.1) wird in der Logik der Fallpauschalenzuordnung in den bisherigen Versionen unter SwissDRG stark gewichtet. Die Fallschwere und der damit verbundene Erlös erhöhen sich in der Regel, weil mit dieser Diagnose ein signifikant höherer Ressourcenverbrauch verbunden ist.

Mittels Antragsverfahren können die Kantone, die Versicherer, der Spitalverband H+ und die FMH bzw. die medizinischen Fachgesellschaften die Entwicklung des Fallpauschalensystems SwissDRG wie auch die Anpassung der Klassifikationskataloge und deren Anwendung beantragen. Bereits 2016 reichten betroffene

\section{Moderne Medizin braucht eine differenzierte} Abgeltung und eine breit abgestützte Datengrundlage.

Fachgesellschaften mit der Unterstützung der FMH einen Antrag zur Aktualisierung der Richtlinie zur Kodierung der Sepsis gemäss der neuen Definition beim BFS ein. Im Mai 2017 bestand unter Vertretern des BFS, der SwissDRG AG und den Delegierten verschiedener betroffener Fachgesellschaften dahingehend Konsens, den Antrag per 2019 umzusetzen.

In der Folge implementierte die SwissDRG AG die Sepsis-3 in der Gruppierungslogik für die SwissDRG-Ver- 
sion 8.0, gültig ab 1.1.2019. Entsprechend misst diese Version der Diagnose «R65.1 SIRS infektiöser Genese mit Organkomplikation» keine Fallschwere bei. Ausschlaggebend sind neu die Diagnose "Sepsis" sowie die behandlungspflichtigen Organdysfunktionen. Von essentieller Bedeutung ist daher die korrelierende Anpassung der gültigen Kodierrichtlinie der Sepsis. Ohne die Kongruenz beider Elemente ist keine aufwandsgerechte Vergütung der Sepsisfälle unter Berücksichtigung der jeweiligen Fallschwere möglich. Auch die datenbasierte Auswertung der Fälle zur Optimierung der Gruppierungssoftware für zukünftige Versionen wird über die Dauer der Inkongruenz verunmöglicht.

\section{Ressourcenmangel beim BFS}

Für den Erlass und die Pflege der Klassifikationssysteme ist das BFS verantwortlich. Es stützt sich dafür auf die Technische Expertenkommission (TEEX), einen beratenden Ausschuss. Die Kommission setzt sich zusammen aus den Partnern der SwissDRG AG: den Versicherern, Vertretern der schweizerischen Konferenz der kantonalen Gesundheitsdirektorinnen und -direktoren (GDK), Delegierten von H+ sowie der FMH. Letztere erreichte innerhalb der betroffenen Fachgesellschaften eine Konsolidation betreffend eine neue Sepsis-Kodierrichtlinie. Aufgrund einer Ressourcenknappheit beim BFS hat die FMH die Initiative zur Abstimmung der Kodierrichtline innerhalb der TEEX ergriffen - damit die Adaption der SwissDRG-Version 8.0 an die klinische Anwendung auch in der Abrechnung eine Abbildung findet.

Trotz des erzielten breiten Konsenses hat das BFS aufgrund des Personalmangels entschieden, die Richtlinie nicht in das Kodierungshandbuch 2019 aufzunehmen. Dies obwohl die Inkohärenz zum Gruppierungssystem bereits bekannt war. Eine Anpassung der Kodierrichtlinie «Sepsis» wurde für 2021 in Aussicht gestellt.

\section{Koordination und Kooperation sind grundlegende Voraussetzungen}

Der Bundesrat fungiert als letzte Instanz zur Genehmigung von Tarifversionen und Kodierrichtlinien. Als letzte Möglichkeit, die unspezifische Zuordnung, eine undifferenzierte Abgeltung und eine unverwertbare Datenerzeugung zu vermeiden, blieb der Weg zum Bundesrat über den Verwaltungsrat der SwissDRG AG. Denn jede geplante Abrechnungsversion muss zusammen mit den vom BFS erstellten Kodierrichtlinien dem Bundesrat zur Genehmigung vorgelegt werden. Der Verwaltungsrat der SwissDRG hat beschlossen, dem Bundesrat die von der FMH und den Fachgesellschaften entworfene Sepsis-Kodierrichtlinie zur Annahme zu empfehlen. Die Zustimmung des Bundesrates zur SwissDRG-Version 8.0 vorausgesetzt, ersetzt die unter https://www.swissdrg.org $\rightarrow$ Akutsomatik $\rightarrow$ Hinweise zur Leistungserfassung $\rightarrow$ PDF Kodierrichtlinie Sepsis publizierte Kodierrichtlinie die im Kodierungshandbuch veröffentlichte Version. So fände zu guter Letzt dank interdisziplinärer Zusammenarbeit - ein klinischer Fortschritt zeitnah Abbildung im Tarifsystem.

\section{Literatur}

1 Weis S, Dickmann P, Pletz M W, Coldewey S M, Gerlach H, Bauer M. Sepsis 2017: Eine neue Definition führt zu neuen Konzepten; Dtsch Arztebl. 2017;114(29-30):A-1424-8.

Die konsolidierte Kodierrichtlinie Sepsis finden Sie unter: https://www.swissdrg.org $\rightarrow$ Akutsomatik $\rightarrow$ Hinweise zur Leistungserfassung $\rightarrow$ PDF Kodierrichtlinie Sepsis 